\title{
Por uma teoria do urbanismo de cunhas verdes
}

\author{
Por una teoría del urbanismo de cuñas verdes
}

\section{For a theory of the urbanism of green wedges}

\author{
Fabiano Lemes de Oliveira \\ fabiano.lemes@polimi.it \\ Politecnico di Milano, DAStU, Milão, Itália
}

\begin{abstract}
Resumo: Este artigo apresenta um modelo de equilíbrio entre urbanização e natureza. Inicialmente, o artigo contextualiza o papel da natureza no enfrentamento de desafios contemporâneos chave, sobretudo relacionados ao enfrentamento das previsões dos severos efeitos negativos consequentes das alterações climáticas, bem como de destruição da natureza por atividades antrópicas ou dela decorrentes. Em seguida oferece aspectos fundamentais da teoria de urbanismo de cunhas verdes e explora a aplicabilidade da ideia em face às problemáticas urbanas brasileiras. Conclui-se que o urbanismo de cunhas verdes, devido a sua particular articulação entre áreas urbanizadas e livres, apresenta uma estrutura efetiva e flexível que pode contribuir para a questão urbana em diferentes domínios, sobretudo na maximização dos benefícios da natureza no seio do tecido urbano.
\end{abstract}

Palavras-chave: planejamento verde; natureza; cidades; tipologia de espaços livres

Resumen: En este artículo se presenta un modelo de equilibrio entre urbanización y naturaleza. Inicialmente, trata del papel de la naturaleza en el enfrentamiento de desafíos contemporáneos clave, sobremodo relacionados al enfrentamiento de los severos efectos negativos consecuentes del cambio climático previstos, así como de destrucción de la naturaleza por actividades antrópicas o derivadas de ella. Luego, ofrece aspectos fundamentales de la teoría de urbanismo de cuñas verdes y explora la aplicabilidad de la idea frente a las problemáticas urbanas brasileñas. Se concluye que el urbanismo de cuñas verdes, debido a su particular articulación entre áreas urbanizadas y libres, presenta una estructura efectiva y flexible que puede contribuir a la cuestión urbana en diferentes dominios, sobre todo en la maximización de los beneficios de la naturaleza en el seno del tejido urbano.

Palabras clave: planificación ecológica; naturaleza; ciudades; tipología de espacios libres.

\begin{abstract}
This paper presents a model of balancing urbanization and nature. Firstly, it presents contextual information about the role of nature in facing key contemporary challenges, in particular related to facing the predicted negative consequences of climate change, as well as the destruction of the natural environment because of or derived from anthropic activities. Subsequently, it introduces fundamental aspects of the theory of green wedge urbanism and explores the applicability of the idea in contributing to tackling Brazilian urban challenges. We concluded that green wedge urbanism, given its particular articulation between urbanised and natural areas, presents an effective and flexible structure that can contribute to the urban question in different domains, above all in the maximization of the benefits of nature throughout the urban fabric.
\end{abstract}

Keywords: green planning; nature; cities; typology of free spaces. 


\section{NATUREZA E CIDADE EM FACE AOS DESAFIOS CONTEMPORÂNEOS}

A necessidade de se acomodar uma crescente população urbana e ao mesmo tempo significativamente reduzir as pressões antrópicas no planeta, além de salvaguardar o capital natural existente, é um dos principais paradoxos da atualidade. Até 2050, espera-se que a população global chegue a nove bilhões de pessoas, das quais $70 \%$ viverão em áreas urbanas (UN, 2018). No Brasil, a população urbana deve exceder $90 \%$. Sabe-se ainda que as cidades são responsáveis por cerca de $80 \%$ das emissões de carbono e que a urbanização se associa à diminuição da vitalidade ecológica do planeta (WWF, 2016). Alguns dos conhecidos riscos associados à questão climática incluem o aumento das temperaturas, aumento do nível do mar, processos simultâneos de desertificação e inundação, maior frequência de eventos climáticos extremos e extinção de flora e fauna. Criar ou adaptar cidades para que possam responder de modo sustentável e resiliente a imperativos habitacionais crescentes, aos possíveis efeitos das mudanças climáticas e que integrem urbanização e natureza de modo efetivo e simbiótico colocam-se como um dos principais desafios contemporâneos.

Neste contexto, significativa atenção internacional tem se dado à importância da natureza nas cidades. Pesquisa sobre os serviços ecossistêmicos - ou seja os benefícios derivados da natureza categorizados em serviços de proteção, regulação, provisão, culturais e suporte - está em alta. Aplicado ao ambiente urbano, e consciente dos potenciais 'desserviços', este entendimento está no cerne das produções sobre infraestrutura verde (BENEDICT; MCMAHON, 2006; EU, 2013; PELLEGRINO; MOURA, 2017), cidades biofílicas (BEATLEY, 2011), re-naturalização das cidades (LEMES DE OLIVEIRA; MELL, 2019) e soluções baseadas na natureza (EU, 2015).

A questão da determinação do ambiente antrópico em relação ao natural data dos primórdios da nossa ocupação do território e permeia a discussão do planejamento das cidades. Contrariamente a visões dicotômicas superficiais de diferenciação absoluta entre os seres humanos e a natureza, a cidade e o campo, cultura e primitivismo ou o artificial e o natural, observa-se dentro da história das ideias urbanísticas nuances integrativas entre uma urbanidade não mediada, como por exemplo a visão metropolitana radical de L. Hilberseimer dos anos 1920, e a quase completa 'naturalidade', tal qual seria viver na cabana primitiva (RYKWERT, 1972). Seja por proximidade e diferenciação em função das necessidades antagônicas de proteção e subsistência como nos primeiros assentamento humanos, seja pela busca ativa da integração entre urbanização e natureza, como no plano de Cerdà para Barcelona, há um arcabouço teórico-histórico importante e ainda relevante a partir do qual se pode procurar reconceituar o modo como pensamos nosso lugar e o de nossas cidades na natureza. O desenvolvimento da Ecologia, no relacionamento de indivíduos e habitats em ecossistemas, bem como a influência do pensamento sistêmico a partir dos anos 1960 abriram um caminho de exploração no planejamento de cidades em que a integração dos sistemas urbanos com aqueles não antrópicos passa a ser não apenas significativo procedimento metodológico, mas também meta de realização. Designing with Nature, de Ian McHARG (1969), bem exemplificou esta busca; seguida a posteriori por publicações como Green Urbanism (BEATLEY, 2000), a coletânea de Waldheim (2006) sobre 
urbanismo da paisagem, Ecological Urbanism de Mostafavi e Doherty (2010) e Urban Ecology de Forman (2014), dentre outros. Meu livro Green Wedge Urbanism (2017) se posiciona nesta vertente de discussão teórica. Sua contribuição fundamental é trazer à luz, a partir de uma reflexão histórica crítica e análise de casos contemporâneos, um modelo de planejamento verde e uma tipologia de espaços livres que pode em muito beneficiar o futuro de nossas cidades e regiões.

\section{URBANISMO DE CUNHAS VERDES}

Cunhas verdes são espaços livres em forma de cunha que penetram o tecido urbano até as áreas centrais, normalmente estreitando-se na medida em que o fazem. A ideia, aplicada à noção de um modelo de planejamento, pressupõe uma articulação particular entre urbanização e espaços livres em que estes se colocam de modo alternado aos espaços edificados. A ideia foi proposta pelo urbanista alemão Rudolf Eberstadt no começo do século XX como o modelo mais adequado para a inclusão sistêmica de áreas verdes no tecido urbano das cidades modernas (LEMES DE OLIVEIRA, 2014). Para Eberstadt, tais cidades teriam sua expansão baseada não em círculos concêntricos como havia sido o caso para a maioria das cidades europeias pré-industriais, mas sim dada ao longo dos eixos viários que radiavam do centro citadino aos subúrbios. Este modo de ocupação do território teria gerado 'vazios' em forma de cunha entre estes eixos de urbanização. Ao invés de considerá-los áreas à espera de edificação, Eberstadt propõe que sejam transformadas em cunhas verdes, trazendo o campo e áreas naturais ao centro das cidades. O modelo, em contraposição a ideia de cinturão verde - que manteria grande parte das áreas verdes distantes de onde a maioria da população urbana vive - teria a capacidade intrínseca, pela sua forma, de trazer a natureza para o cerne da vida urbana. Os principais benefícios do modelo de cunhas verdes desde o princípio de sua conceptualização seriam a distribuição equitativa de áreas verdes para todos residentes e proximidade a elas, a reconexão com o campo, zoneamento adequado separando áreas industriais de residenciais, e a criação de canais que facilitariam a entrada de ar puro nas cidades e remoção do ar poluído.

A ideia teve grande difusão internacional durante o século $X X$, com propagação teórica por nomes como Patrick Abercrombie, autor do County of London Plan 1943 e do Greater London Plan 1944 (LEMES DE OLIVEIRA, 2015), além de projetos por toda Europa, a então União Soviética, Austrália, Japão, dentre outros. No Brasil, a ideia se difundiu principalmente através do Plano de Avenidas de Prestes Maia para São Paulo e do plano de Alfred Agache para o Rio de Janeiro, ambos de 1930.

A retomada da ideia se dá com ênfase em vários planos urbanísticos contemporâneos, notadamente nos países escandinavos e na Europa continental. O desenvolvimento recente da ideia de cunhas verdes, para além das funções iniciais, inclui preocupações afinadas com à crescente evidência dos benefícios do contato com a natureza e como resposta aos desafios globais contemporâneos, como a sustentabilidade e resiliência urbana. Mas, quais seriam os benefícios específicos do modelo de cunhas verdes em face a estes desafios? 
O modelo de cunhas verdes proporciona, intrinsecamente pela sua forma, maior proximidade aos espaços verdes comparados a modelos de desenvolvimento urbano tradicionais (Fig.1). Isso significa que os serviços ecossistêmicos são trazidos para perto das pessoas, para as proximidades de onde elas vivem, trabalham, se locomovem e se recreiam. Sabe-se que o uso de espaços livres é impactado diretamente pela proximidade e acessibilidade a ele pelos residentes (LEMES DE OLIVEIRA, 2019; VRIES et al., 2003). Através da adequada distribuição de cunhas verdes, isso pode ser garantido. Por exemplo, cidades que implantaram e consolidaram o modelo ao longo das últimas décadas vêem que a maioria dos seus habitantes reside no raio de 1.000 metros de uma cunha verde, como é o caso de Estocolmo, com 97\%, Helsinki, 91\%, e Copenhagen, $80 \%$. Ainda, devido a sua forma, as cunhas verdes podem expandir-se, ou mesmo se retrair, na medida em que a cidade passa por processos de expansão ou encolhimento (LEMES DE OLIVEIRA, 2017).

Figura 1: Diagrama do modelo de cunhas verdes.

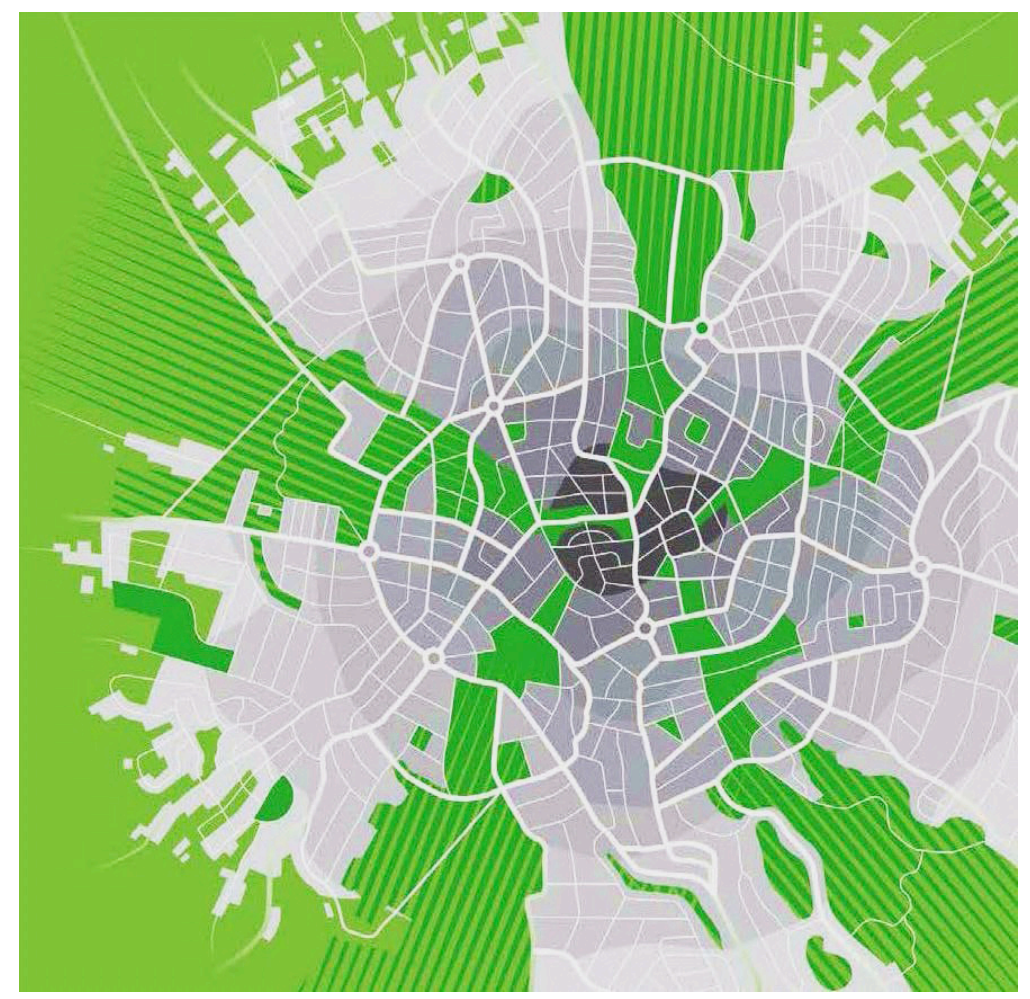

Fonte: LEMES DE OLIVEIRA, 2014

A conexão entre a cidade e o campo, ou áreas naturais vizinhas, têm sido constantemente outro benefício do modelo. As cunhas verdes podem se configurar como elementos de conexão da paisagem tanto para seres humanos como para outras espécies. $\mathrm{O}$ comprometimento do domínio ecológico em paisagens fragmentadas é bem documentado. Johnson e Munshi-South (2017) sumarizam este efeito em espécies predominantemente terrestres mostrando como tendem, dentre outros efeitos, à redução do número de indivíduos e ao empobrecimento da variedade genética. As cunhas verdes podem maximizar as oportunidades para corredores ecológicos contínuos, por sua vez aumentando a presença 
de flora e fauna mesmo nas áreas urbanas mais centrais. O parque central de Helsinki, com mais de $11 \mathrm{~km}$ de extensão, é um exemplo de cunha verde que faz a conexão direta do centro com áreas naturais na franja urbana, proporcionando paisagens contínuas e integradas (Fig. 2).

Figura 2: Parque Central de Helsinque e algumas de suas funções - cultural, recreativa, de preservação ecológica e agricultura urbana.
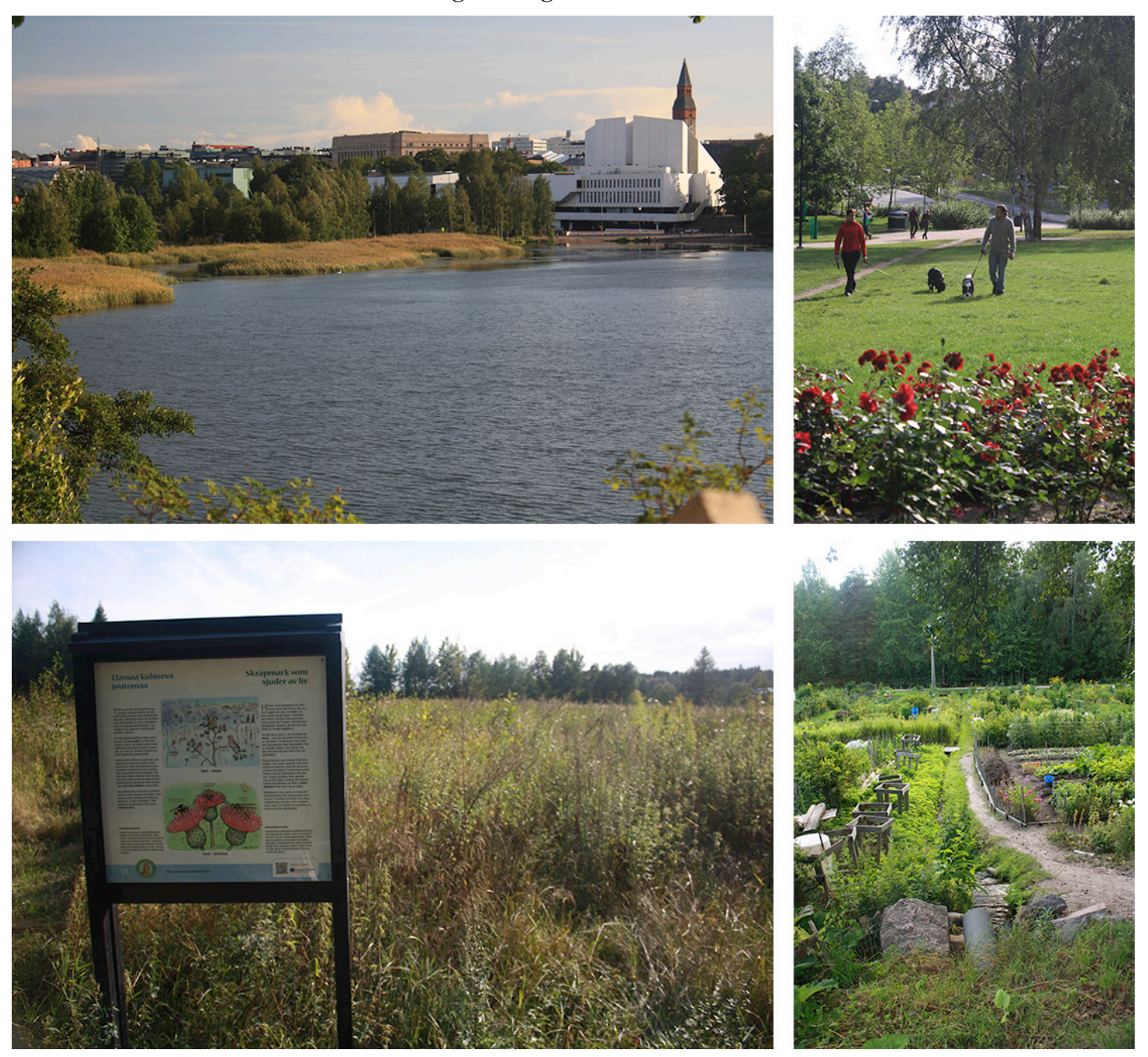

Fonte: o autor.

A impermeabilização do solo urbano e sua expansão em direção ao campo levam ao aumento das temperaturas nas cidades; da poluição sonora, do ar e luminosa; impactando ainda na saúde física e mental dos residentes. Com o prospecto das mudanças climáticas, espera-se que as temperaturas globais se elevem sobremaneira. Efeitos estes que já se manifestam. Por exemplo, considera-se que o verão britânico de 2018 foi um dos mais quentes desde que se coletam dados. Adelaide, na Australia, bateu o recorde de temperatura chegando a mais de 46 graus em janeiro de 2019. O Brasil também neste ano de 2019 vem enfrentando um dos seus verões mais inclementes. O fenômeno da UHI (Urban Heat Island / Ilha de Calor Urbana - ICU), ou seja a observação de temperaturas mais altas em áreas 
urbanas comparadas a áreas fora das cidades, tende a crescer tendo em vista a previsão de aumento da urbanização e das temperaturas globais. Cunhas verdes podem ajudar a reduzir estas temperaturas e sensação térmica, tanto pelo sombreamento proporcionado pelas áreas verdes, como pelos fenômenos das evapo-transpiração e adequada circulação do ar. Freiburg, na Alemanha, faz efetivo uso de uma cunha verde regional para amenizar as temperaturas urbanas. A cidade controla a ocupação do solo e proíbe a verticalização em porção da cunha verde regional ao longo do vale do Rio Dreisam com o objetivo de permitir os fluxos de ar entre a cidade e a Floresta Negra, o que reduz drasticamente as temperaturas no verão no centro da cidade, comparadas com outras áreas não afetadas por este sistema de ventos.

Similarmente, as cunhas verdes são eficazes aliadas no combate à poluição do ar, que é hoje o maior risco ambiental mundial. Sua área vegetada contribui na purificação do ar e de modo geral as cunhas tem sido empregadas, como no caso de Freiburg, também como dutos de ventilação que retiram a poluição das áreas urbanas, inserindo ao invés fluxos de ar fresco.

Espaços verdes de larga escala, como as cunhas verdes, oferecem a possibilidade de acomodação de várias funções e atividades para diversos grupos sociais, etários e com interesses distintos. Estas multiplicidades colaboram por sua vez para taxas de uso altas (SUGIYAMA et al., 2010). Espaços bem usados, normalmente são melhor tratados e 'adotados' pela população. Outros usos mais gerais se relacionam, por exemplo, com a preservação ambiental, manutenção de áreas permeáveis ao longo dos leitos fluviais e como parte de estratégias de drenagem urbana sustentável, dentre outras.

Considerando-se os benefícios intrínsecos expostos acima, Green Wedge Urbanism definiu uma teoria baseada em dez princípios diretores para a aplicação em cidades e regiões. Sua implementação foi pensada tanto como definição de uma estrutura principal dentro de infraestruturas verdes com espaços variados e granulares, bem como através da sua implementação tipológica. Estes princípios são: a busca de uma relação equilibrada entre áreas edificadas e natureza, conexão entre cidade e campo, continuidade das cunhas verdes, maximização do acesso e uso público, o planejamento positivo e o projeto de lugares de alta qualidade, a integração sistêmica e um plano, a inclusão dos serviços ecossistêmicos, permeabilidade das bordas das cunhas verdes e conexões cruzadas, uma aproximação multi-escalar e flexibilidade da forma.

\section{DESAFIOS DA URBANIZAÇÃO BRASILEIRA E CUNHAS VERDES}

Apesar de que historicamente as cidades brasileiras tenham contado com significativa integração com áreas naturais, os processos de urbanização desencadeados com a modernidade e o espraiamento contemporâneo tendem a diminuir e fragmentar áreas verdes em cidades, bem como, na medida em que se expandem, reduzir as áreas de campo ou naturais. A urbanização mal ou nada planejada tem seus efeitos nefastos manifestos, dentre outros, em enchentes, sequias, deslizes de terra, escassez de áreas verdes e azuis de 
qualidade, poluição, desequilíbrios ecológicos e reforço de desigualdades em seus mais amplos sentidos. Tais problemas são visíveis tanto nas metrópoles brasileiras como nas cidades de menor porte (ANGEOLETTO; SANTOS, 2015; LEMES DE OLIVEIRA; MELL, 2019). Uma mudança de visão em direção ao pensamento sistêmico de integração entre sistemas antrópicos e naturais é necessária. Cidades médias são loci privilegiados para ação imediata. Frequentemente, contam ainda com área urbana disponível, possiblidade de efetiva integração no planejamento urbano-rural a nível regional e potencial de implementação de diretrizes de uso do solo que áreas mais consolidadas encontrariam mais limitadas por conta de processos de dependência da trajetória. A rápida urbanização das cidades médias do Brasil, onde vivem cerca de 52 milhões de brasileiros (ANGEOLETTO et al., 2016), impõe a essas cidades a pronta adoção de soluções que conjuguem a conservação da biodiversidade com o incremento da qualidade de vida humana (ANGEOLETTO, 2018; RUMBLE et al., 2019).

Esta integração sistêmica bem como o crescimento do entendimento do papel da re-naturalização das cidades em enfrentar nossos desafios são quadros conceituais e práticas cujos benefícios são múltiplos e atravessam distintas escalas. A forma urbana, neste sentido, joga um papel importante. Ela pode facilitar ou dificultar tais processos de integração dos sistemas socioculturais e ecológico-ambientais. Assim, o urbanismo de cunhas verdes - na sua particular articulação entre áreas urbanizadas e livres, cidade e adjacências - apresenta uma estrutura das mais efetivas e flexíveis para maximizar a concepção ou transformação de cidades em equilíbrio com a natureza.

\section{REFERÊNCIAS}

ANGEOLETTO, Fabio. A busca por cidades saudáveis. Estudos Avançados, v. 32, n. 93, p. 255-259, 2018. ANGEOLETTO, Fabio; SANTOS, Jeater W.M.C. Los biólogos brasileños no habitan en el planeta ciudad: por qué es urgente formar ecólogos urbanos. Revista Espaço Acadêmico, v. 14, n. 165, p. 74-82, 2015.

ANGEOLETTO, Fábio; SANTOS, Jeater W.M.C.; SANZ, Juan et al. Tipología socio-ambiental de las ciudades medias de Brasil: aportes para un desarrollo urbano sostenible. Urbe - Revista Brasileira de Gestão Urbana, v. 8, n. 2, p. 48-66, 2016.

BEATLEY, Timothy. Green urbanism: learning from European cities. Washington: Island Press, 2000.

BEATLEY, TIMOTHY. Biophilic Cities: integrating Nature into Urban Design and Planning. Washington: Island Press, 2011.

BENEDICT, Mark A.; MCMAHON, Edward. Green Infrastructure: linking Landscapes and Communities. Washington: Island Press, 2006.

EU - European Comission. Building a Green Infrastructure for Europe. Luxembourg, 2013.

EU - European Comission. Towards an EU Research and Innovation policy agenda for Nature-Based Solutions \& Re-Naturing Cities Luxembourg, 2015. (Final Report of the Horizon 2020 Expert Group on 'Nature Based Solutions and Re-Naturing Cities').

FORMAN, Richard T.T. Urban Ecology: Science of Cities. Cambridge: Cambridge University Press, 2014.

JOHNSON, Marc T.J.; MUNSHI-SOUTH, Jason. Evolution of life in urban environments. Science, v. 358, n. 6363, p. 1-11, 2017. 
LEMES DE OLIVEIRA, Fabiano. Green wedges: origins and development in Britain. Planning Perspectives, v. 29, n. 3, p. 357-379, 2014.

LEMES DE OLIVEIRA, Fabiano. Abercrombie's green-wedge vision for London: the County of London Plan 1943 and the Greater London Plan 1944. Town Planning Review, v. 86, n. 5, p. 495-518, 2015.

LEMES DE OLIVEIRA, Fabiano. Green Wedge Urbanism: History, Theory and Contemporary Practice. London/ New York: Bloomsbury, 2017.

LEMES DE OLIVEIRA, Fabiano. Towards a Spatial Planning Framework for the Re-Naturing of Cities. In: LEMES DE OLIVEIRA, Fabiano; MELL, Ian (Orgs.). Planning Cities with Nature: Theories, Strategies and Methods. Amsterdam: Springer Nature, 2019. p. 81-95.

LEMES DE OLIVEIRA, Fabiano; MELL, Ian (Orgs.). Planning Cities with Nature: Theories, Strategies and Methods. Amsterdam: Springer Nature, 2019.

McHARG, Ian. Design with nature. New York: Natural History Press, 1969.

MOSTAFAVI, Mohsen; DOHERTY, Gareth. Ecological Urbanism. Karlsruhe: Lars Müller, 2010.

PELLEGRINO, Paulo; MOURA, Newton Becker (Orgs.). Estratégias para uma Infraestrutura Verde. Barueri: Manole, 2017.

RYKWERT, Joseph. On Adam's house in Paradise: the idea of the primitive hut in architectural history. New York: Museum of Modern Art, 1972.

RUMBLE, Heather; ANGEOLETTO, Fabio.; CONNOP, Stuart. et al. Understanding and Applying Ecological Principles in Cities. In: LEMES DE OLIVEIRA, Fabiano; MELL, Ian (Orgs.). Planning Cities with Nature: Theories, Strategies and Methods. Amsterdam: Springer Nature, 2019. p. 217-234. DOI:10.1007/978-3-03001866-5.

SUGIYAMA, Takemi; FRANCIS, Jacinta; MIDDLETON, Nicholas J. et al. Associations Between Recreational Walking and Attractiveness, Size, and Proximity of Neighborhood Open Spaces. American Journal of Public Health, v. 100, n. 9, p. 1752-1757, 2010.

UN - United Nations. World Urbanization Prospects: The 2018 Revision. New York, 2018.

VRIES, Sjerp de; VERHEIJ, Robert; GROENEWEGEN, Peter; SPREEUWENBERG, Peter. Natural environments - healthy environments? An exploratory analysis of the relationship between greenspace and health. Environment and Planning A, v. 35, 1717-1731, 2003.

WALDHEIM, Charles. The Landscape Urbanism Reader. New York: Princeton Architectural Press, 2006.

WWF. Living Planet Report: Risk and resilience in a new era. Gland, 2016. Disponível em: http:/ / awsassets. panda.org/downloads/lpr_2016_full_report_low_res.pdf Acesso em: 25 set. 2019.

Data de submissão: 05/ mar./ 2019

Data de aceite: $01 /$ jul./2019 